\title{
A First Approach to Contact-Based Biometrics for User Authentication
}

\author{
Athanasios Vogiannou ${ }^{1,2}$, Konstantinos Moustakas ${ }^{2}$, Dimitrios Tzovaras $^{2}$, \\ and Michael G. Strintzis ${ }^{1,2}$ \\ 1 Electrical \& Computer Engineering Department, \\ Aristotle University of Thessaloniki \\ 2 Informatics and Telematics Institute, CERTH
}

\begin{abstract}
This paper presents the concept of contact-based biometric features, which are behavioral biometric features related to the dynamic manipulation of objects that exist in the surrounding environment. The motivation behind the proposed features derives from activity-related biometrics and the extension of them to activities involving objects. The proposed approach exploits methods from different scientific fields, such as virtual reality, collision detection and pattern classification and is applicable to user authentication systems. Experimental results in a dataset of 20 subjects show that the introduced features comprise a very efficient and interesting approach in the research of biometric features.
\end{abstract}

\section{Introduction}

Biometrics have recently gained significant attention from researchers while they have been rapidly developed for various commercial applications ranging from access control against potential impostors to the management of voters to ensure no one votes twice [12]. These systems require reliable personal recognition schemes to either confirm or determine the identity of an individual requesting their services. A number of approaches have been described in the past to satisfy the different requirements of each application such as unobtrusiveness, reliability, permanence, etc.

Biometric methods are categorized to physiological and behavioral [3, depending on the type of used features. Physiological biometrics are based on biological measurements and inherent characteristics of each human. The fingerprint is a typical example of physiological biometrics which is widely used in law enforcement for identifying criminals 4]. Other recent applications are based on identification with the iris [5] or the face [6].

On the other hand, behavioral biometrics are related to specific actions and the way that each person executes them. The most known example of behavioral biometrics is gait recognition [7]. Behavioral biometrics are the newest technology in biometrics and they have yet to be researched in detail. They are supposed to be less reliable than physiological biometrics, however they are less obtrusive and simpler to implement 38 . 
Both types of biometric features are based on human characteristics of everyday life. One such interesting characteristic is the hand and the way people used it to manipulate objects. Although significant amount of research has been performed on various aspects of dynamic hand gestures 910], such features have not been employed for biometrics yet.

The present paper introduces contact-based biometric features and investigates the feasibility of such features in user authentication applications. The presented concept derives from the simple observation that every person handles the objects of the surrounding environment quite differently. For example, the action of picking up a glass or holding a knife depends on the physiological characteristics of each person and the way that this person is used to manipulate objects. Contact biometrics belong to the general category of behavioral biometrics and can also been thought as a specialization of activity-related biometrics [117.

The general overview of the framework is given in the section 2 , while sections 3. 4] and 5] describe in detail the setup, the feature extraction and the authentication modules of the proposed approach respectively. Experimental results are provided in section [6] and the conclusions are drawn in section 7 .

\section{Overview}

Although humans interact with objects in various ways, the present work concentrates on the hand as it is the major human part for object manipulation. It is important to point out that contact-based features of the hand are not the same as hand biometrics which have already been employed for human recognition 12. Although certain hand characteristics, such as the size of the palm or the length of the fingers, have an effect in the way humans manipulate objects, contact-based biometrics are primarily concerned with the behavioral features and the dynamics of the specific action. In particular, except from the gesture posture of the hand, contact based features include the information about the collisions between the hand and the object.

In summary the proposed scheme consists of four major parts:

- Setup of a 3D virtual environment

- Registration of the user's hand and the objects in the virtual environment

- Extraction of features during an action performed by the user using an object

- Final classification and authentication

The 3D environment and the registration of the objects in virtual environment is necessary because collision detection algorithms can only be used with knowledge of the 3D geometry of each object. Based on this setup, the next step is to extract different types of features and produce the final authentication result.

\section{3D Environment Setup and Model Registration}

The most important, and at the same time difficult, task of the registration procedure is the $3 \mathrm{D}$ reconstruction of the user's hand. In the proposed method, the 
hand is modeled as a set of 5 fingers connected to the palm, which is represented by a simple rectangle (Figure1(a)). Each finger has 4 Degrees of Freedom (DOF) and consists of 3 phalanxes which are modeled as simple ellipsoids. Other 3 DOF describe the orientation of the whole hand.

For the registration of the hand, the CyberGlove ${ }^{\circledR}$ (www.immersion.com) device was employed. The CyberGlove ${ }^{\circledR}$ (Figure 1(b)) provides the angles between the phalanxes of the hand, and therefore it is possible to reconstruct a 3D representation of the hand. Note, that the virtual representation of the hand is not perfectly accurate because the size of the fingers and the phalanxes are not known. This inaccuracy is considered as noise and does not significantly affect the results.

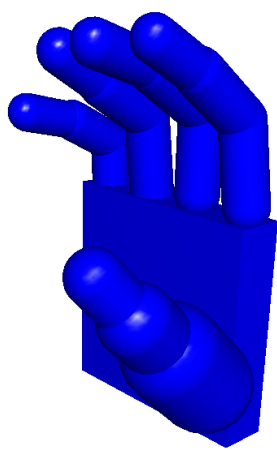

(a)

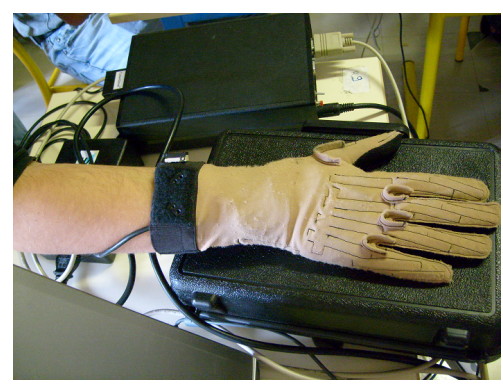

(b)

Fig. 1. The 3D representation of the hand (a) and the CyberGlove ${ }^{\circledR}$ (b)

The objects of the environment can be registered using computer vision techniques for object tracking 13. Since object tracking is out of the scope of this paper, a simple color based tracker [14] was implemented. However, it is not absolutely necessary to have an accurate representation of the object in the virtual environment. Regarding rigid objects, which are the most common case, the geometry of each object can be simplified using a priori information. This simplification is possible as the real shape of each object is usually not directly related to the way it is handled. For example, a glass can be represented by a simple cylinder since the user grabs only the outer surface of the glass.

\section{Feature Extraction}

The classification features of contact-based biometrics are twofold. From one hand there is the feature set of the hand posture (static, user oriented) and on the other the collision information (dynamic, user-object interaction). The first set is related to the hand posture and consists of the data extracted from the CyberGlove ${ }^{\circledR}$ while the second one is based on collision detection algorithms 
and the information that we can retrieve from them. Although collision features are indeed related to the hand posture, the transformation between these two feature spaces is unknown and impossible to define for general shapes due to the algorithmic nature of collision detection methods and the dynamic position of each object.

\subsection{Hand Posture Features}

Any representation of the hand posture can be linearly defined by the angles produced by the CyberGlove ${ }^{\circledR}$ device. Therefore, these angles constitute a feature space of the hand posture. Figure 2 displays the position and the orientation of the joints and the respective measured angles in the hand. Every DOF of each joint corresponds to a feature. The final feature $\boldsymbol{F}_{a}$ vector is a 23-dimensional vector consisting of

$$
\boldsymbol{F}_{a}=\left\{\boldsymbol{W}_{0}, \boldsymbol{W}_{1}, \ldots, \boldsymbol{W}_{22}\right\}
$$

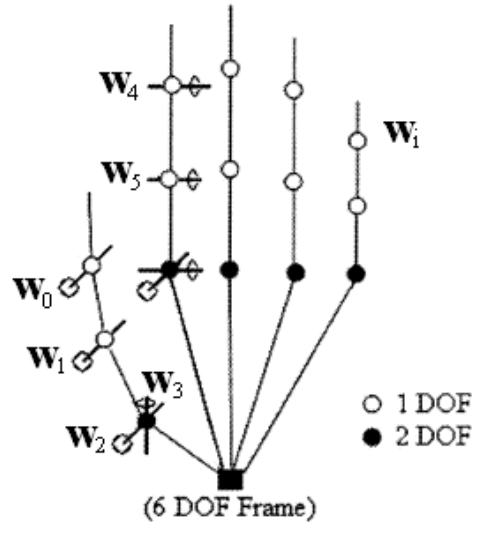

(a)

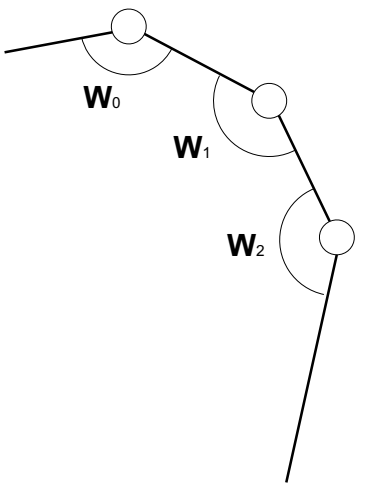

(b)

Fig. 2. Posture Features. Each DOF of the joints between the phalanxes and the palm corresponds to a feature. Figure (a) illustrates the measured angles for all the hand while figure (b) focuses on a single finger.

\subsection{Collision Features}

The collision features consist of any information that can be acquired by employing state-of-the-art algorithms for proximity queries. These include penetration depth [15], closest distance [1617], contact points etc. The literature in the field is vast and there are numerous algorithms to accurately perform queries in realtime. The interested reader is directed to [151617181920|2122] for further details. For the purposes of the presented work, the algorithms for rigid convex objects [2319] of the software package SOLID (http://www.dtecta.com/) were used. 


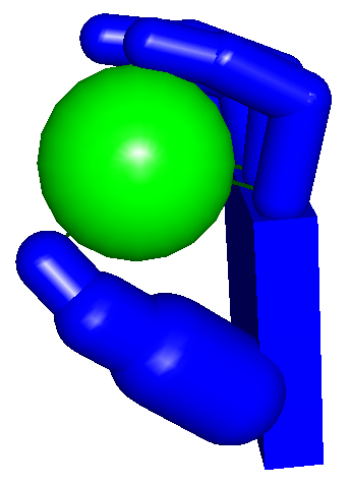

(a)

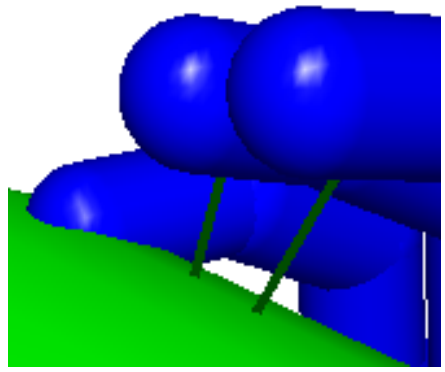

(b)

Fig. 3. Collision Features. Either the closest distance or the penetration depth is used, depending on the collision state between each finger and the object.

Proximity queries are performed between the object and every finger of the user's hand. Each query refers to either of two states, collision or no collision between the two virtual shapes. For example penetration depth can only be calculated when two objects intersect since it is always 0 otherwise. However, in a user-object interaction scheme it is necessary to continuously produce discriminant feature samples. Thus, any proximity query as a single feature would not provide adequate information to a classifier.

In order to overcome this difficulty, the combination of the penetration depth and the closest distance is used, depending on the collision state, to define the feature space. The penetration depth and the closest distance are usually described as $3 \mathrm{D}$ vectors in virtual simulations. However, in our case it is preferred to describe them as the pair of points $\left(\mathbf{p}_{\text {finger }}, \mathbf{p}_{\text {object }}\right)$, one on the finger and the other one on the object, that define the respective vector $\mathbf{v}=\mathbf{p}_{\text {finger }}-\mathbf{p}_{\text {object }}$. This way the $3 \mathrm{D}$ position of each finger affects the values of the feature vector, while $\mathbf{v}$ would only describe the relative direction which is usually similar even for different fingers. The $3 \mathrm{D}$ vectors are represented in the local coordinate system of the hand. Let $\mathbf{p d}_{k}$ and $\mathbf{c d}_{k}$ denote the points of the penetration depth and the closest distance respectively where $k$ is either a finger or the object. The feature sample $f_{e}(i, O)$ for finger $e$ and object $O$ on the $i$-th frame is

$$
f_{e}(i, O)=\left\{\begin{array}{l}
\left(\mathbf{p d}_{e}, \mathbf{p d}_{O}\right), \quad \text { if e and O collide } \\
\left(\mathbf{c d}_{e}, \mathbf{c d}_{O}\right), \text { if e and } \mathrm{O} \text { do not collide }
\end{array}\right.
$$

Figure 3 displays the closest distance between two fingers and the surface of a $3 \mathrm{D}$ object. The final collision feature vector $F=\bigcup_{e}\left\{f_{e}\right\}$ is formed using the collision information from all the 5 fingers and is a 30 -dimensional vector.

The feature vectors of both collision and angle data are calculated during an object-related action at every frame update of the virtual environment. Note that in a realistic application we would also need an activity recognition module to detect object-related actions in a free environment [24]. However, the presented 
work focuses on the evaluation of contact-biometrics and not in the general setup of a specific application. Therefore, it is considered that the extracted feature data refer only to a specific object-related action.

\section{Classification and User Authentication}

In general, authentication systems involve an enrollment stage where the system calculates and stores a template of the subject's feature measurement. For most biometric features, this is a single feature vector. However, in the case of the presented method, the enrolment sessions generate a significant number of samples due to the high sampling frequency $(90 \mathrm{smples} / \mathrm{sec})$ of the CyberGlove ${ }^{\circledR}$ and the dynamics of the action. The set of enrollment samples is used for training a classifier so that it recognizes impostors from genuine users.

More specifically, let $F_{i}$ denote the training set of feature samples of subject $s_{i}$. The enrollment sample $f_{i}{ }^{e}$ is selected as the average feature of the enrollment session. Then, the feature sets of genuine user $G_{i}$ and impostor users $I_{i}$ are generated by

$$
f \in G_{i}: f=a-f_{i}{ }^{e} \quad \text { where } \quad a \in F_{i}
$$

and

$$
f \in I_{i}: f=a-f_{i}^{e} \quad \text { where } \quad a \in F_{k \neq i}
$$

The above 2-class classification problem is solved with Support Vector Machines (SVMs) in the proposed method. In particular, a SVM $M_{i}$ is trained for each subject $s_{i}$ so that it separates $G_{i}$ from $I_{i}$.

When a user claims to be the subject $s_{i}$, the system samples the respective feature vector $f$ and calculates $f_{d}=f-f_{i}{ }^{e}$. If $f_{d}$ is classified in $G_{i}$, the user $s_{i}$ is authenticated. Otherwise the user is considered to be an impostor.

\section{Experimental Results}

The experimental setup includes one testing action that is performed by 20 subjects. In particular, the right hand of each user and a glass were registered in the virtual environment for the action notated as "grabbing the glass". Each person performed the action 10 times which produced around 1000 feature samples in total for each subject.

In the following, the results are presented in two parts. The first part evaluates the distinctiveness while the second part the performance of the proposed authentication approach. The results are also separated according to the type of features, i.e. only collision features, only angle features and the combination of the two, and according to the type of the kernel used with the SVM.

\subsection{Authentication Distinctiveness}

Table 1 displays the False Rejection and False Acceptance rates for the 8 test sessions of the 20 subjects. All the sessions were performed with the same calibration of the glove for each subject, i.e. the subjects did not put off the glove 
Table 1. FRR and FAR for 20 subjects

\begin{tabular}{|c|c|c|c|c|c|c|}
\hline Kernel Type & \multicolumn{3}{|c|}{ Linear } & \multicolumn{3}{c|}{ RBF } \\
\hline Features & Posture & Collision & Combined & Posture & Collision & Combined \\
\hline FRR & $0.01 \%$ & $0.5 \%$ & $0 \%$ & $0 \%$ & $0.1 \%$ & $0 \%$ \\
\hline FAR & $0 \%$ & $0.08 \%$ & $0 \%$ & $0 \%$ & $0 \%$ & $0 \%$ \\
\hline
\end{tabular}

between sessions. From the acquired samples, the first session was used for the enrollment and the rest were used for testing.

The results demonstrate the high distinctiveness of the proposed features since FRR and FAR are practically 0. Although these results confirm our assumption, there is also the concept of consistency and the reliability of the measurements which is evaluated in the next section.

\subsection{Authentication Performance}

The term performance used here is generally defined in [3]. More specifically, what is of concern in our case is the consistency and the accuracy of the measurements. In order to evaluate the performance of the proposed approach, data acquisition were performed with two different calibrations. More specifically, each subject performed another set of 10 sessions but in the meantime it put off the glove and put it on again. No restrictions or guidelines were given on how to wear the glove at either of the session sets.

Table 2 displays the results. The efficiency of the method was reduced, when compared to the first data-set (Figure1). This is caused by the difference in the way that each subject put on the glove at the second calibration, introducing this way a significant amount of undefined noise. Nevertheless, the combination of the features is very robust, especially for the FRR. This fact illustrates the high authentication efficiency of the proposed approach in the presence of noise.

Table 2. FRR and FAR for 20 subjects with different calibrations of the glove

\begin{tabular}{|c|c|c|c|c|c|c|}
\hline Kernel Type & \multicolumn{3}{|c|}{ Linear } & \multicolumn{3}{c|}{ RBF } \\
\hline Features & Posture & Collision & Combined & Posture & Collision & Combined \\
\hline FRR & $2.5 \%$ & $4.2 \%$ & $0.6 \%$ & $2.3 \%$ & $3.13 \%$ & $0.6 \%$ \\
\hline FAR & $4.3 \%$ & $10.76 \%$ & $4.5 \%$ & $4.3 \%$ & $5.8 \%$ & $4.5 \%$ \\
\hline
\end{tabular}

\section{Conclusions and Future Work}

In this paper we presented the novel contact-based biometric features that are related to activity-related biometrics and include the dynamic characteristics of interactions with objects. The results show that the proposed method can achieve very high rates of authentication performance and therefore comprises a very interesting approach for further research in activity-related biometrics. The proposed method can be integrated along with other types of features in a user authentication system so as to improve the overall efficiency. 


\section{Acknowledgements}

This work was supported by the EU funded ACTIBIO IST STREP (FP7215372).

\section{References}

1. Qazi, F.A.: A survey of biometric authentication systems. In: Security and Management, pp. 61-67 (2004)

2. Xiao, Q.: Security issues in biometric authentication. In: Information Assurance Workshop, IAW 2005, June 2005, pp. 8-13 (2005)

3. Jain, A., Ross, A., Prabhakar, S.: An introduction to biometric recognition. IEEE Transactions on Circuits and Systems for Video Technology 14(1), 4-20 (2004)

4. Maltoni, D., Maio, D., Jain, A.K., Prabhakar, S.: Handbook of Fingerprint Recognition. Springer, Heidelberg (2003)

5. Chowhan, S., Shinde, G.: Iris biometrics recognition application in security management, vol. 1, pp. 661-665 (2008)

6. Chang, K.I., Bowyer, K.W., Flynn, P.J.: An evaluation of multimodal $2 \mathrm{~d}+3 \mathrm{~d}$ face biometrics. IEEE Trans. Pattern Analysis and Machine Intelligence 27(4), 619-624 (2005)

7. Boulgouris, N., Chi, Z.: Gait recognition using radon transform and linear discriminant analysis. IEEE Transactions on Image Processing 16(3), 731-740 (2007)

8. Delac, K., Grgic, M.: A survey of biometric recognition methods. In: Electronics in Marine, 2004. Proceedings Elmar 2004. 46th International Symposium, pp. 184-193 (2004)

9. Moustakas, K., Strintzis, M., Tzovaras, D., Carbini, S., Bernier, O., Viallet, J., Raidt, S., Mancas, M., Dimiccoli, M., Yagci, E., Balci, S., Leon, E.: Masterpiece: Physical Interaction and 3D Content-based Search in VR Applications. IEEE Multimedia 13(3), 92-100 (2006)

10. Tzovaras, D., Nikolakis, G., Fergadis, G., Malasiotis, S., Stavrakis, M.: Design and Implementation of Haptic Virtual Environments for the Training of the Visually Impaired. IEEE Transactions on Neural Systems and Rehabilitation Engineering 12(2), 266-278 (2004)

11. Kale, A., Cuntoor, N., Chellappa, R.: A framework for activity specific human recognition. In: International Conference on Acoustics, Speech and Signal Processing, Orlando, FL (2002)

12. Kukula, E., Elliott, S.: Implementation of hand geometry: an analysis of user perspectives and system performance. IEEE Aerospace and Electronic Systems Magazine 21, 3-9 (2006)

13. Yilmaz, A., Javed, O., Shah, M.: Object tracking: A survey. ACM Comput. Surv. 38(4) (2006)

14. Ren, Y., Chua, C.S., Ho, Y.K.: Color based tracking by adaptive modeling. In: 7th International Conference on Control, Automation, Robotics and Vision, ICARCV 2002., vol. 3, pp. 1597-1602 (2002)

15. Kim, Y.J., Lin, M.C., Manocha, D.: Incremental penetration depth estimation between convex polytopes using dual-space expansion. IEEE Transactions on Visualization and Computer Graphics 10(2), 152-163 (2004)

16. Larsen, E., Gottschalk, S., Lin, M., Manocha, D.: Fast distance queries with rectangular swept sphere volumes, vol. 4, pp. 3719-3726 (2000) 
17. Lin, M.C., Canny, J.F.: A fast algorithm for incremental distance calculation, pp. 1008-1014 (1991)

18. Lin, M.C., Gottschalk, S.: Collision detection between geometric models: A survey. In: IMA Conference on Mathematics of Surfaces, pp. 37-56 (1998)

19. van den Bergen, G.: Collision Detection in Interactive 3D Environments. Morgan Kaufmann, San Francisco (2003)

20. Ericson, C.: Real-Time Collision Detection. Morgan Kaufmann, San Francisco (2004)

21. Thomas, F., Torras, C.: 3D collision detection: A survey. Computers and Graphics 25, 269-285 (2001)

22. Teschner, M., Kimmerle, S., Zachmann, G., Heidelberger, B., Raghupathi, L., Fuhrmann, A., Cani, M.P., Faure, F., Magnetat-Thalmann, N., Strasser, W.: Collision detection for deformable objects. In: Eurographics State-of-the-Art Report (EG-STAR), pp. 119-139. Eurographics Association (2004)

23. van den Bergen, G.: Efficient collision detection of complex deformable models using AABB trees. Journal on Graphics Tools 2 (1997)

24. Gavrila, D.M.: The visual analysis of human movement: a survey. Computer Vision and Image Understanding 73(1), 82-98 (1999) 